\title{
Neurogenesis Makes a Crucial Contribution to the Neuropathology of Alzheimer's Disease
}

\author{
John K. Young* \\ Professor Emeritus, Department of Anatomy, Howard University College of Medicine, Washington, DC, USA
}

Accepted 15 August 2020

\begin{abstract}
One unexplained feature of Alzheimer's disease (AD) is that the lateral entorhinal cortex undergoes neurodegeneration before other brain areas. However, this brain region does not have elevated levels of amyloid peptides in comparison with undamaged regions. What is the cause of this special vulnerability of the entorhinal cortex? One special feature of the lateral entorhinal cortex is that it projects to newborn neurons that have undergone adult neurogenesis in the dentate gyrus of the hippocampus. Neurogenesis is abnormal in human AD brains, and modulation of neurogenesis in experimental animals influences the course of AD. This complex process of neurogenesis may expose axon terminals originating from neurons of the entorhinal cortex to a unique combination of molecules that can enhance toxic effects of amyloid. Retrograde degeneration of neurons with axons terminating in the dentate gyrus provides a likely explanation for the spatial patterns of neuronal cell death seen in AD. Specialized astrocytes in the dentate gyrus participate in adult neurogenesis and produce fatty acid binding protein7 (FABP7). These FABP7+ cells undergo an aging-related mitochondrial pathology that likely impairs their functions. This age-related abnormality may contribute to the impairment in neurogenesis seen in aging and Alzheimer's disease. Also, a compromised function of these astrocytes likely results in local elevations of palmitic acid, iron, copper, and glucose, which all enhance the toxicity of amyloid peptides. Treatments that modulate neurogenesis or diminish the production of these toxic substances may prove more successful than treatments that are solely aimed at reducing the amyloid burden alone.
\end{abstract}

Keywords: Alzheimer's disease, astrocytes, copper, entorhinal cortex, FABP7, Gomori-positive, neurogenesis

The modern era of understanding Alzheimer's disease (AD) began some thirty years ago with the identification of the proteins and enzymes that contribute to the formation of amyloid plaques found in the brains of sufferers from AD. Since then, details of the structure of amyloid- $\beta$ protein precursor $(\mathrm{A} \beta \mathrm{PP})$, its incorporation into amyloid plaques, and its cleavage into harmful peptides by $\beta$ - and $\gamma$-secretases have been identified. Inherited mutations in these proteins cause the early onset, or familial, form of AD.

\footnotetext{
${ }^{*}$ Correspondence to: John K. Young, PhD, 9902 Oleander Avenue, Vienna, VA 22181, USA. Tel.: +1 703255 3591; E-mail: jyoung@howard.edu.
}

The amyloid cascade hypothesis proposes that a disturbed function of these proteins also causes the more widespread, sporadic form of $\mathrm{AD}$ [1].

One means by which A $\beta P P$ and amyloid cleavage products cause neuronal pathologies is by the induction of abnormalities in tau. Tau is a microtubule-associated protein that is hyperphosphorylated in $\mathrm{AD}$, leading to the formation of so-called neurofibrillary tangles in neurons. The appearance of tau-induced neurofibrillary tangles is more closely correlated with neuronal death and cognitive declines than the presence of amyloid plaques. Moreover, changes in tau have been reported to precede any significant elevations in cleavage products of amyloid 
$\left(A \beta_{42}\right)$ that are measurable in the cerebrospinal fluid. These types of data have led to suggestions that treatments that target abnormal tau may be more helpful than treatments aimed at reducing amyloid alone (reviewed in [2]).

Some additional problems with the amyloid cascade hypothesis are apparent. One problem is that brains of many individuals who are cognitively normal can have an amyloid plaque burden similar to that found in demented patients [2]. Also, damaged neurons containing neurofibrillary tangles are first found in the entorhinal cortex, whereas amyloid plaques first seem to appear at some distance from this site in the association cortices of the temporal lobe [2, $3]$. One widely accepted explanation for these discrepancies is that soluble fragments of $\mathrm{A} \beta P P(\mathrm{~A} \beta$ oligomers), and not amyloid plaques themselves, are the cause of damage to synapses and other structures in $\mathrm{AD}$. Thus, the establishment of plaques alone may not be the precipitating event for the disorder. Extracellular amyloid oligomers, rather than plaques, may bind to neurons to cause damage $[1,4]$.

In spite of these plausible explanations, it still seems puzzling that initial damage in $\mathrm{AD}$ is confined to specific brain structures, whereas A $\beta P P$ seems to be widely distributed throughout the brain. The hypothesis expressed in this paper contends that it is the toxic combination of $A \beta P P$ and other substances involved in adult neurogenesis within the dentate gyrus of the hippocampus that accounts for the patterns of damage in AD brain. Axons that terminate near newborn neurons in the dentate gyrus may be especially vulnerable to the disease process (see below).

A second problem with the amyloid cascade hypothesis that has recently arisen is that clinical treatments that reduce the amyloid burden in the brain by $18-36 \%$ have failed to slow the cognitive decline in AD patients (reviewed in [4]).

A recent paper may provide some explanation for the disappointing results of these clinical trials of $\mathrm{AD}$ patients. In a transgenic rat model of $\mathrm{AD}$, treatment with scyllo-inositol reduced the amyloid plaque burden in the brain by $35 \%$ but failed to restore all cognitive faculties to normal [5]. Also, this reduction in amyloid failed to diminish tau-associated neurofibrillary tangles in the lateral entorhinal cortex, nor did it improve the disconnect between the lateral entorhinal cortex and the dentate gyrus. This interruption of communication between the dentate gyrus and lateral entorhinal cortex makes an important contribution to disturbances in memory and behavior.
This study showed that a reduction of the amyloid burden in rats was insufficient to correct the neurological and behavioral defects characteristic of AD. This might explain why clinical trials that reduce the amyloid burden in humans have also failed to slow the degradation of cognitive function. A disturbed function of the entorhinal cortex seems to persist, in spite of lowered levels of amyloid. It would appear that damage to the perforant pathway between the lateral entorhinal cortex and dentate gyrus occurs unusually early in the disease process, when amyloid levels are still low, or else this pathway is unusually sensitive to toxic effects of amyloid [5]. But what would cause this heightened sensitivity?

Researchers examining the early degeneration of the lateral entorhinal cortex have reported that levels of amyloid peptides in the lateral entorhinal cortex are not higher than in other, less affected regions $[6,7]$. What, then, accounts for the early degeneration of the lateral entorhinal cortex? One proposed explanation for this discrepancy is that a higher metabolic rate of lateral entorhinal cortex neurons might make these neurons more sensitive to toxic effects of amyloid or $A \beta$ [6].

However, many neurons throughout the brain exhibit similar signs of an elevated metabolic rate and do not degenerate in AD. The mesencephalic trigeminal nucleus, for example, contains neurons with a very high levels of cytochrome oxidase, a marker for elevated oxidative metabolism [8]. This nucleus does not appear to be affected in $\mathrm{AD}$, although other brainstem nuclei like the raphe nucleus or the locus coeruleus do show early signs of degeneration $[3,9]$. What else might be responsible for degeneration of these brainstem nuclei?

A recent study, using a transgenic mouse model of $\mathrm{AD}$, has shown that connectivity between the raphe nucleus and locus coeruleus with the hippocampus becomes interrupted during the course of the disease [10]. Thus, one possible explanation for the degeneration of these brainstem nuclei is that their axon terminals within the hippocampus experience damage that causes a retrograde degeneration of axons and cell bodies. This same explanation may apply to the lateral entorhinal cortex.

Neurons of the lateral entorhinal cortex of rodent brains are unique in that they provide the main afferent connections to newly born neurons that have undergone neurogenesis in the adult brain [11]. The highly unusual process of adult neurogenesis has also been reported to take place in the dentate gyrus of the human hippocampus [12-17]. There has been some 
controversy about whether the neurogenesis observed in rodent hippocampi really is also present in the human brain, but recent methods of improved tissue preservation of human brain tissue have confirmed that markers for neurogenesis are indeed present in the human hippocampus $[15,17]$. If lateral entorhinal cortex neurons of the human brain also innervate newborn neurons of the dentate gyrus, as in the mouse brain, then lateral entorhinal neurons indeed occupy a unique niche in the human brain. It may not be a coincidence that entorhinal cortex neurons directly connected to newly born neurons are the first to degenerate in $\mathrm{AD}$.

What is the evidence for an involvement of adult neurogenesis in the disease process of AD? Hippocampal neurogenesis is decreased in brains from $\mathrm{AD}$ patients [15]. In mouse models of $\mathrm{AD}$, transgenic for abnormal human proteins, genetic manipulations can induce a blockade of neurogenesis. This decrease in neurogenesis worsens cognitive deficits. On the other hand, stimulation of neurogenesis in a mouse model of AD lessens cognitive deficits $[18,19]$. These results may relate to the stimulatory effects of neurogenesis upon memory processes and a compensation for the disruption of hippocampal function seen in AD.

Another point is that A $\beta P P$ itself appears to influence neurogenesis, so that abnormalities in A $\beta P P$ function could result in impaired neurogenesis [18, 20]. In light of these findings, some researchers have suggested that adult neurogenesis may have a role in the pathogenesis of $\mathrm{AD}[21,22]$.

It may be that toxic effects of amyloid peptides may first be exerted upon axons terminating upon newborn neurons and perhaps extend in a retrograde fashion to damage neuronal somas of the lateral entorhinal cortex [23].

Adult neurogenesis involves the division of neuronal cell precursors, their differentiation into glial or neuronal cell lineages, and their incorporation into the circuitry of the hippocampus. What aspects of these complex events may exert a toxic interaction with amyloid or amyloid peptides that could affect synapses from fibers arising from the lateral entorhinal cortex?

In macaque and rat brains, specialized astrocytes immunoreactive for fatty acid binding protein 7 (FABP7) are in close contact with newly born neurons of the dentate gyrus and appear to represent stem cells from which these new neurons have originated (Fig. 1). The phenotype of these cells appears to recapitulate that of FABP7-containing radial glial cells of

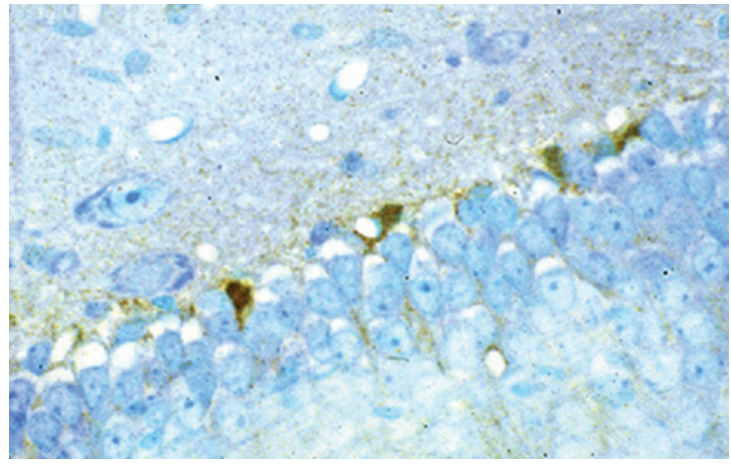

Fig. 1. Astrocytes immunoreactive for FABP7 (brown stain) are found within the granule cell layer of the dentate gyrus of the hippocampus of adult rats. See [26] for staining methodology.

the embryonic cortex that differentiate into neurons [24]. The hippocampus is not the only brain site at which these specialized astrocytes can be detected. FABP7+ astrocytes are also found in the hypothalamus, the habenula, and the dorsal medulla, sites at which much more modest amounts of adult neurogenesis take place [24-27].

FABP7+ astrocytes in the hypothalamus have been found to undergo an aging-related pathological process, in which degenerating mitochondria become engulfed within lysosomes to form socalled Gomori-positive cytoplasmic granules [26]. This mitochondrial pathology is also detectable at the precise locations of FABP7+ astrocytes in the dentate gyrus, habenula, subventricular zone, and dorsal medulla ([24] versus [27,31]). Thus, it appears that an aging-related mitochondrial degeneration is a widespread feature of FABP7+ astrocytes that participate in adult neurogenesis.

What is the cause of this astrocyte pathology? One likely explanation is that these cells have an elevated uptake and metabolism of fatty acids. Mitochondrial oxidation of fatty acids is known to exert particularly damaging effects upon astrocyte mitochondria [24, 28].

An aging-related pathology of FABP7+ astrocytes might reasonably be expected to reduce their ability to divide and mature into neurons and could contribute to the aging-related decline in neurogenesis seen in rodent brains. This could also be a factor in the decreased neurogenesis seen in brains of humans with AD.

There are also a number of ways by which this astrocyte pathology could enhance toxic effects of amyloid. FABP7+ astrocytes regulate levels of palmitic acid in the brain [29]. Addition of palmitic 
acid to $\mathrm{A} \beta \mathrm{PP}$ enhances its processing into amyloidogenic and toxic peptides, so any disturbance of the regulation of palmitic acid by degenerating FABP7+ astrocytes could enhance amyloid toxicity [30].

Also, the Gomori-positive cytoplasmic granules of FABP7+ astrocytes contain high levels of heme-linked copper and iron, originating from metal-containing mitochondrial enzymes [31]. Copper binds to amyloid peptides, reduces the clearance of amyloid, and appears capable of enhancing toxic effects of amyloid; iron also enhances toxic effects of amyloid [32, 33]. Since about $3.5 \%$ of hippocampal glia turn over and die every year, local release of these metals from senescent FABP7+ astrocytes could have toxic effects [34].

A specific isoform of a cholesterol-binding protein called ApoE4 drastically enhances the risk for AD [35]. ApoE4 is mainly produced by astrocytes; its deleterious effects in AD have mainly been ascribed to ApoE-related effects on the clearance of amyloid peptides. However, in mice, ApoE4 has also been shown to reduce dendritic spines upon newly born neurons in the hippocampus, leading to a reduced connectivity between the lateral entorhinal cortex and the hippocampus [36]. Thus, effects of ApoE4 upon neurogenesis, as well as effects of ApoE4 upon amyloid degradation, could be related to its association with a greatly increased risk for AD.

Intracellular heme and its modulating enzyme, heme oxygenase, exert complex effects upon the production of cholesterol and ApoE in astrocytes [37]. Possibly, the presence of heme in FABP7+ astrocytes could alter the local production of ApoE isoforms and could lead to alterations in both the degradation of amyloid and in the maturation of newborn neurons in the dentate gyrus. This hypothesis could be tested by examination of Gomori-positive astrocytes in vitro to see if they produce altered amounts of ApoE.

Another feature of $\mathrm{AD}$ is an association with diabetes mellitus, which increases the risk for dementia $[32,38]$. The precise reasons for this association are uncertain, although many mechanisms have been proposed. For one thing, diabetes causes a reduction in adult neurogenesis. Also, high blood levels of glucose induce the formation of advanced glycation end products within the brain. These products, plus the receptors for these end products, are abundant in glial cells and can provoke alterations in the production of $\mathrm{A} \beta$ peptides. Also, glycation of amyloid enhances its toxicity [39]. Some involvement of Gomori-positive astrocytes in the interaction between diabetes and $\mathrm{AD}$ may be indicated by the fact that these cells possess high capacity GLUT2 type glucose transporter proteins. These GLUT2 transporters enable cells to have an elevated glucose uptake and an increased responsiveness to changes in blood glucose concentrations compared to other brain cells [40]. Indeed, in AD, Gomori-positive astrocytes of the human hippocampus have elevated levels of advanced glycation end products in their cytoplasm [41]. Any disturbance in the function of Gomori-positive astrocytes in diabetes could thus logically be expected to exacerbate the abnormalities seen in AD.

Much of the data about FABP7+ astrocytes and their role in neurogenesis stems from studies of the rodent and macaque brains. Can this information be extended with some degree of confidence to the human brain? The presence of mRNA for FABP7 has been reported in both adult and fetal human brain samples. Fetal brain samples have been obtained from individuals suffering from the human medical condition, trisomy 21, or Down's syndrome.

The presence of FABP7 mRNA in Down's syndrome brains is of particular interest because this condition is associated with an increased risk for the development of $\mathrm{AD}$ and dementia. One logical explanation for this association is the presence of the gene for amyloid precursor protein $(A P P)$ on chromosome 21 ; the presence of an extra chromosome 21 leads to an increased expression of A $\beta P P$ and enhanced deleterious effects of amyloid and amyloid oligomers [1]. However, in Down's syndrome brains, the expression of FABP7 is also increased, due to the presence on chromosome 21 of a protein that regulates the transcription of the FABP7 gene [42]. The authors of this paper speculated that increased levels of FABP7 during development could contribute to the neurological abnormalities found in this syndrome. This speculation is supported by a recent report that astrocytes with an enhanced expression of FABP7 exert negative effects upon neuronal survival in vitro [43]. These data provide increased support for the concept that FABP7+ astrocytes in the human brain could contribute to the neuronal abnormalities in AD. Thus far, however, immunocytochemistry for FABP7+ astrocytes in the human hippocampus has not yet been performed.

The presence of Gomori-positive astrocytes in the human hippocampus has, in contrast, been reported in two publications [41, 44]. Also, Gomori-positive astrocytes have been demonstrated in frozen sections of the human hypothalamus collected for an unrelated study some years ago (Fig. 2) [45]. These 


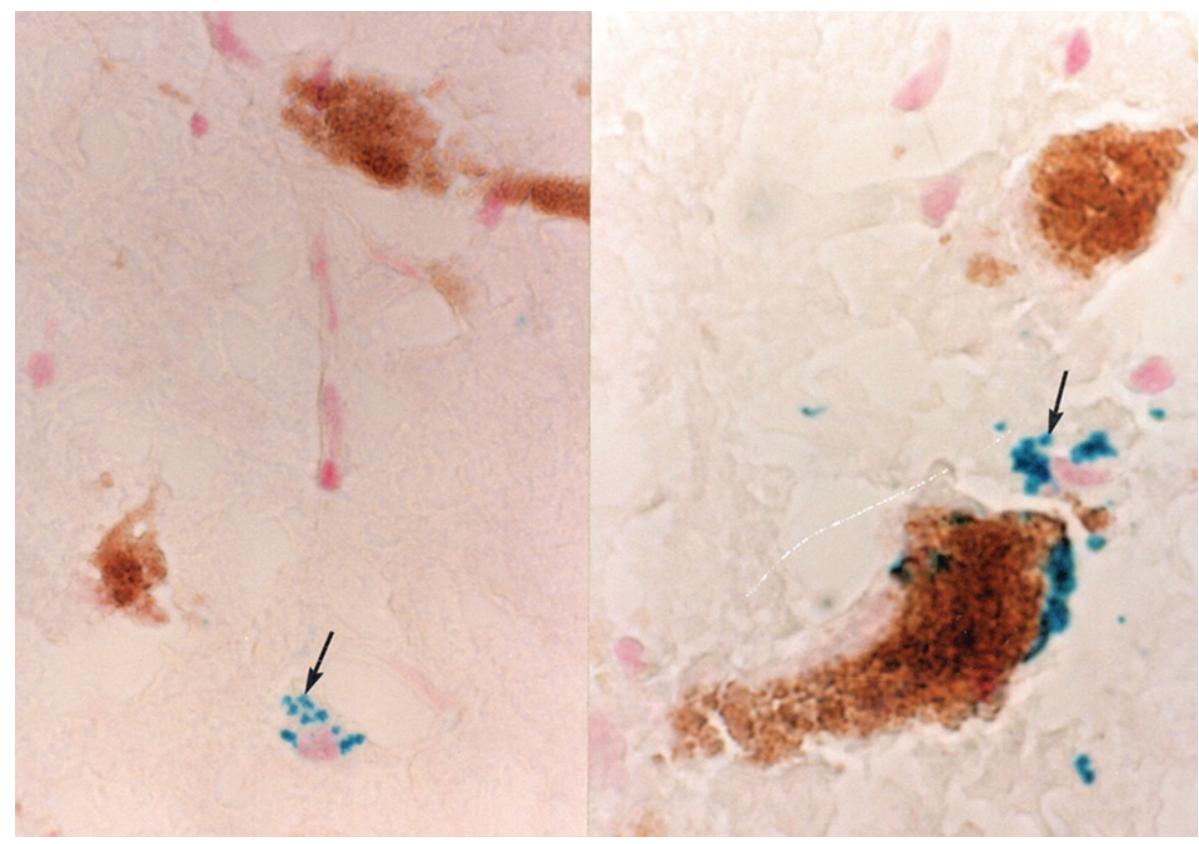

Fig. 2. Frozen section of the human hypothalamus, stained to demonstrate iron via the Perl's reaction and counterstained with neutral red to illustrate cell nuclei (see [40] for Perl's stain methodology). Blue iron-rich cytoplasmic granules (arrows) are found in a Gomori-positive astrocyte (left side of figure). On the right side of the figure, a Gomori-positive astrocyte is in close contact with a catecholaminergic neuron filled with neuromelanin.

cells are commonly adjacent to catecholaminergic neurons filled with neuromelanin, a metabolic byproduct of catecholamines. A similar situation exists in the rat hypothalamus, where Gomoripositive astrocytes can be found in contact with catecholaminergic neurons [46]. These data indicate that Gomori-positive astrocytes of the human brain have many features in common with those of the rat brain.

If Gomori-positive astrocytes are abundant in both the human and rat hypothalamus, it would be logical to expect that the presence of these cells could also lead to hypothalamic neuropathology, as well as to hippocampal neuropathology, in $\mathrm{AD}$. Plaques, tangles, and a disturbed neuronal function in the hypothalamus are early features of $\mathrm{AD}$, but the question of whether these abnormalities are directly related to Gomori-positive astrocytes remains to be investigated [47].

Other features of neurogenesis, independent of FABP7+ astrocytes, could also contribute to the pathology of $\mathrm{AD}$ and explain its focus in the lateral entorhinal cortex. The apparent connection between neurogenesis and $\mathrm{AD}$, however, provides a novel framework for an understanding of the features of $\mathrm{AD}$, such as the specific neuroanatomical distributions of damaged neurons, the relationship between
$\mathrm{AD}$ and aging of glia, and the interrelationships between AD, diabetes, ApoE isoforms, and Down's syndrome.

Strategies that would diminish age-related abnormalities in astrocytes participating in neurogenesis, such as drugs that reverse aging-associated mitochondrial impairments, low fat diets, diets rich in polyunsaturated fatty acids, or a reduced dietary intake of copper, may prove useful in limiting the development of AD pathology [48, 49, 50, 51]. Another approach would be to increase the differentiation of FABP7+ astrocytes into mature neurons, by administering drugs that increase neurogenesis [52]. This would logically deplete the pool of FABP7+ neural precursor cells and reduce the harmful molecules associated with them. An alternative method of increasing neurogenesis may be to increase physical exercise, which has been reported to increase rates of neurogenesis and reduce the risk for $\mathrm{AD}$ [53].

\section{CONFLICT OF INTEREST}

The author declares that he has no competing interests. 


\section{REFERENCES}

[1] Selkoe DJ, Hardy J (2016) The amyloid hypothesis of Alzheimer's disease at 25 years. EMBO Mol Med 8, 595608.

[2] Morris GP, Clark IA, Vissel B (2018) Questions concerning the role of amyloid- $\beta$ in the definition, aetiology and diagnosis of Alzheimer's disease. Acta Neuropathol 136, 663-689.

[3] Braak H, Thal DR, Ghebremedhin E, Del Tredici K (2011) Stages of the pathologic process in Alzheimer disease: Age categories from 1 to 100 years. J Neuropathol Exp Neurol 70, 960-969.

[4] Ricciarelli R, Fedele E (2017) The amyloid cascade hypothesis in Alzheimer's disease: It's time to change our mind. Curr Neuropharmacol 15, 926-935.

[5] Morrone CD, Bazzigaluppi P, Beckett TL, Hill ME, Koletar MM, Stefanovic B, McLaurin J (2020) Regional differences in Alzheimer's disease pathology confound behavioural rescue after Amyloid- $\beta$ attenuation. Brain 143, 359-373.

[6] Khan UA, Liu L, Provenzano FA, Diego E Berman DE, Caterina P Profaci CP, Sloan R, Mayeux R, Duff KE, Small SA (2014) Molecular drivers and cortical spread of lateral entorhinal cortex dysfunction in preclinical Alzheimer's disease. Nat Neurosci 17, 304-311.

[7] Stranahan AM, Mattson MP, Alexis M (2010) Selective vulnerability of neurons in layer II of the entorhinal cortex during aging and Alzheimer's disease. Neural Plast 2010, 108190.

[8] Hevner RF, Liu S, Wong-Riley MT (1995) A metabolic map of cytochrome oxidase in the rat brain: Histochemical, densitometric and biochemical studies. Neuroscience 65, 313-342.

[9] Iatrou A, Kenis G, Rutten BPF, Lunnon K, van den Hove DLA (2017) Epigenetic dysregulation of brainstem nuclei in the pathogenesis of Alzheimer's disease: Looking in the correct place at the right time? Cell Mol Life Sci 74, 509-523.

[10] Jeon SG, Kim YJ, Kim KA, Mook-Jung I, Moon M (2018) Visualization of altered hippocampal connectivity in an animal model of Alzheimer's disease. Mol Neurobiol 55, 7886-7899.

[11] Woods NI, Vaaga CE, Chatzi C, Adelson JD, Collie MF, Perederiy JV, Tovar KR, Westbrook GL (2018) Preferential targeting of lateral entorhinal inputs onto newly integrated granule cells. J Neurosci 38, 5843-5853.

[12] Curtis MA, Low VF, Faull RLM (2012) Neurogenesis and progenitor cells in the adult human brain: A comparison between hippocampal and subventricular progenitor proliferation. Dev Neurobiol 72, 990-1005.

[13] Dennis CV, Suh LS, Rodriguez ML, Kril JJ, Sutherland GT (2016) Human adult neurogenesis across the ages: An immunohistochemical study. Neuropathol Appl Neurobiol 42, 621-638.

[14] Lazarov O, Hollands C (2016) Hippocampal neurogenesis: Learning to remember. Prog Neurobiol 138-140, 1-18.

[15] Moreno-Jiménez EP, Flor-García M, Terreros-Roncal J, Rábano A, Cafini F, Pallas-Bazarra N, Ávila J, LlorensMartín M (2019) Adult hippocampal neurogenesis is abundant in neurologically healthy subjects and drops sharply in patients with Alzheimer's disease. Nat Med 25, 554-560.

[16] Teixeira CM, Pallas-Bazarra N, Bolós M, Terreros-Roncal J, Ávila J, Llorens-Martín M (2018) Untold new beginnings: Adult hippocampal neurogenesis and Alzheimer's disease. J Alzheimers Dis 64(s1), S497-S505.
[17] Tobin MK, Musaraca K, Disouky A, Shetti A, Bheri A, Honer WG, Kim N, Dawe RJ, Bennett DA, Arfanakis K, Lazarov O (2019) Human hippocampal neurogenesis persists in aged adults and Alzheimer's disease patients. Cell Stem Cell 24, 974-982.e3.

[18] Hollands C, Tobin MK, Hsu M, Musaraca K, Yu TS, Mishra R, Kernie SG, Lazarov O (2017) Depletion of adult neurogenesis exacerbates cognitive deficits in Alzheimer's disease by compromising hippocampal inhibition. Mol Neurodegener 12, 64.

[19] Richetin K, Leclerc C, Toni N, Gallopin T, Pech S, Roybon L, Rampon C (2015) Genetic manipulation of adult-born hippocampal neurons rescues memory in a mouse model of Alzheimer's disease. Brain 138, 440-455.

[20] Caillé I, Allinquant B, Dupont E, Bouillot C, Langer A, Müller U, Prochiantz A (2004) Soluble form of amyloid precursor protein regulates proliferation of progenitors in the adult subventricular zone. Development 131, 2173-2181.

[21] Choi SH, Tanzi RE (2019) Is Alzheimer's disease a neurogenesis disorder? Cell Stem Cell 25, 7-8.

[22] Mu Y, Gage FH (2011) Adult hippocampal neurogenesis and its role in Alzheimer's disease. Mol Neurodegener 6, 85.

[23] Alobuia WM, Xia W, Vohra BP (2013) Axon degeneration is key component of neuronal death in amyloid- $\beta$ toxicity. Neurochem Int 63, 782-789.

[24] Young JK, Heinbockel T, Gondre-Lewis MJ (2013) Astrocyte fatty acid binding protein-7 is a marker for neurogenic niches in the rat hippocampus. Hippocampus 23, 14761483.

[25] Sousa-Ferreira L, Pereirade Almeida L, Cavadas C (2014) Role of hypothalamic neurogenesis in feeding regulation. Trends Endocrinology Metab 25, 80-88.

[26] Young JK, Baker JH, Muller T (1996) Immunoreactivity for brain fatty acid binding protein in Gomori-positive astrocytes. Glia 16, 218-226.

[27] Pushkar Y, Robison G, Sullivan B, Fu SX, Kohne M, Jiang W, Rohr S, Lai B, Marcus MA, Zakharova T, Zheng W (2013) Aging results in copper accumulations in glial fibrillary acidic protein-positive cells in the subventricular zone. Aging Cell 12, 823-832.

[28] Schmidt SP, Corydon TJ, Pedersen CB, Bross P, Gregersen N (2010) Misfolding of short-chain acyl-CoA dehydrogenase leads to mitochondrial fission and oxidative stress. $\mathrm{Mol}$ Genet Metab 100, 155-162.

[29] Owada Y, Abdelwahab SA, Kitanaka N, Sakagami H, Takano H, Sugitani Y, Sugawara M, Kawashima H, Kiso Y, Mobarakeh JI, Yanai K, Kaneko K, Sasaki H, Kato H, Saino-Saito S, Matsumoto N, Akaike N, Noda T, Kondo $\mathrm{H}$ (2006) Altered emotional behavioral responses in mice lacking brain-type fatty acid-binding protein gene. Eur $J$ Neurosci 24, 175-187.

[30] Bhattacharyya R, Barren C, Kovacs DM (2013) Palmitoylation of amyloid precursor protein regulates amyloidogenic processing in lipid rafts. J Neurosci 33, 11169-11183.

[31] Sullivan B, Robison G, Pushkar Y, Young JK, Manaye KF (2017) Copper accumulation in rodent brain astrocytes: A species difference. J Trace Elements Med Biol 39, 6-13.

[32] Chung JY, Kim H-S, Song J (2018) Iron metabolism in diabetes-induced Alzheimer's disease: A focus on insulin resistance in the brain. Biometals 31, 705-714.

[33] Huat TJ, Camats-Perna J, Newcombe EA, Valmas N, Kitazawa M, Medeiros R (2019) Metal toxicity links to Alzheimer's disease and neuroinflammation. $J$ Mol Biol 431, 1843-1868. 
[34] Spalding KL, Bergmann O, Alkass K, Bernard S, Salehpour M, Huttner HB, Boström E, Westerlund I, Vial C, Buchholz BA, Possnert G, Mash DC, Druid H, Frisén J (2013) Dynamics of hippocampal neurogenesis in adult humans. Cell 153, 1219-1227.

[35] Belloy ME, Napolioni V, Greicius MD (2019) A quarter century of APOE and Alzheimer's disease: Progress to date and the path forward. Neuron 101, 820-838.

[36] Tensaouti Y, Stephanz EP, Yu TS, Kernie SG (2018) ApoE regulates the development of adult newborn hippocampal neurons. eNeuro 5, e0155-18.2018 1-15.

[37] Vaya J, Schipper HM (2007) Oxysterols, cholesterol homeostasis, and Alzheimer disease. J Neurochem 102, 1727-1737.

[38] Pugazhenthi S, Qin L, Reddy PH (2017) Common neurodegenerative pathways in obesity, diabetes, and Alzheimer's disease. Biochim Biophys Acta Mol Basis Dis 1863, 10371045.

[39] Li XH, Du LL, Cheng XS, Jiang X, Zhang Y, Lv BL, Liu R, Wang JZ, Zhou XW (2013) Glycation exacerbates the neuronal toxicity of $\beta$-amyloid. Cell Death Dis, 4, e673.

[40] Young JK, McKenzie JC (2004) GLUT2 immunoreactivity in Gomori-positive astrocytes of the hypothalamus. $J$ Histochem Cytochem 52, 1519-1524.

[41] Takeda A, Yasuda T, Miyata T, Goto Y, Wakai M, Watanabe M, Yasuda Y, Horie K, Inagaki T, Doyu M, Maeda K, Sobue G (1995) Advanced glycation end products colocalized with astrocytes and microglial cells in Alzheimer's disease brain. Acta Neuropathol 95, 555-558.

[42] Sánchez-Font MF, Bosch-Comas A, Gonzàlez-Duarte R, Marfany G (2003) Overexpression of FABP7 in Down syndrome fetal brains is associated with PKNOX1 gene-dosage imbalance. Nucleic Acids Res 31, 2769-2777.

[43] Killoy KM, Harlan BA, Pehar M, Vargas MR (2020) FABP7 upregulation induces a neurotoxic phenotype in astrocytes. Glia, doi: 10.1002/glia.23879.

[44] Ohm TG, Jung E, Schnecko A (1992) A subpopulation of hippocampal glial cells specific for the zinc-containing mossy fibre zone in man. Neurosci Lett 145, 181-184,
[45] Young JK, Stanton GB (1994) A three-dimensional reconstruction of the human hypothalamus. Brain Res Bull 35, 323-327,

[46] Young JK, McKenzie JC, Baker JH (1990) Association of iron-containing astrocytes with dopaminergic neurons of the arcuate nucleus. J Neurosci Res 25, 204-213.

[47] Ishii M, Iadecola C (2015) Metabolic and non-cognitive manifestations of Alzheimer's disease: The hypothalamus as both culprit and target of pathology. Cell Metab, 22, 761776.

[48] Boneva NB, Kikuchi M, Minabe Y, Yamashima T (2011) Neuroprotective and ameliorative actions of polyunsaturated fatty acids against neuronal diseases: Implication of fatty acid-binding proteins (FABP) and G protein-coupled receptor 40 (GPR40) in adult neurogenesis. J Pharmacol Sci 116, 163-172.

[49] Borten O, Liberman A, Tuchweber B, Chevalier S, Ferland G, Schipper HM (2004) Effects of dietary restriction and metal supplementation on the accumulation of iron-laden glial inclusions in the aging rat hippocampus. Biogerontology 5, 81-88.

[50] Gomes AP, Price NL, Ling AJ, Moslehi JJ, Montgomery MK, Rajman L, White JP, Teodoro JS, Wrann CD, Hubbard BP, Mercken EM, Palmeira CM, de Cabo R, Rolo AP, Turner N, Bell EL, Sinclair DA (2013) Declining NAD(+) induces a pseudohypoxic state disrupting nuclear-mitochondrial communication during aging. Cell 155, 1624-1638.

[51] Solfrizzi V, Panza F, Frisardi V, Seripa D, Logroscino G, Imbimbo BP, Pilotto A (2011) Diet and Alzheimer's disease risk factors or prevention: The current evidence. Expert Rev Neurother 11, 677-708.

[52] Nakano-Kobayashi A, Awaya T, Kii I, Sumida Y, Okuno Y, Yoshida S, Sumida T, Inoue H, Hosoya T, Hagiwara M (2017) Prenatal neurogenesis induction therapy normalizes brain structure and function in Down syndrome mice. Proc Natl Acad Sci U S A 114, 10268-10273.

[53] Cass SP (2017) Alzheimer's disease and exercise: A literature review. Curr Sports Med Rep 16, 19-22. 